\title{
INVESTIGATION OF LOW-SIDELOBE BEAMPATTERN CONTROLLING METHODS FOR ACOUSTIC TRANSMITTING ARRAY OF UNDERWATER VEHICLES
}

\author{
Zhengyao He, Ph.D.., \\ Qiang Shi, M.S. \\ Shaoxuan Wu, M.S. \\ Institute of Acoustic Engineering, Northwestern Polytechnical University
}

\begin{abstract}
In underwater unmanned vehicles, complex acoustic transducer arrays are always used to transmitting sound waves to detect and position underwater targets. Two methods of obtaining low-sidelobe transmitting beampatterns for acoustic transmitting arrays of underwater vehicles are investigated. The first method is the boundary element model optimization method which used the boundary element theory together with the optimization method to calculate the driving voltage weighting vector of the array. The second method is the measured receiving array manifold vector optimization method which used the measured receiving array manifold vectors and optimization method to calculate the weighting vector. Both methods can take into account the baffle effect and mutual interactions among elements of complex acoustic arrays. Computer simulation together with experiments are carried out for typical complex arrays. The results agree well and show that the two methods are both able to obtain a lower sidelobe transmitting beampattern than the conventional beamforming method, and the source level for each transmitting beam is maximized in constraint of the maximum driving voltage of array elements being constant. The effect of the second method performs even better than that of the first method, which is more suitable for practical application. The methods are very useful for the improvement of detecting and positioning capability of underwater unmanned vehicles.
\end{abstract}

Keywords: underwater unmanned vehicles, detecting and positioning, acoustic transducer, complex array, low-sidelobe beamforming

\section{INTRODUCTION}

To achieve good system performance of the active sonar in underwater unmanned vehicles, the complex acoustic transducer array is always used to radiate sound waves. Good transmitting beampattern such as a low sidelobe level and high transmitting source level is always expected, thus the transmitting sound energy can be focused in the concerned direction, and the long-range object can be detected with a relatively lower transmitting sound energy[1]. In the research of the radiated acoustic field of compact transducer arrays, the mutual interactions among elements must be considered[2,3,4]. The interactions become more significant when the number of elements increases, the distance among elements is smaller and the sound frequency is lower. In the complex array of acoustic transmitting transducers, the array is small in volume with compact elements, and needed to transmit broadband acoustic signal. Consequently, the interactions among elements in the array are definitely significant. On the other side, the radiated acoustic field of the array may be greatly influenced by the baffle with a certain impedance boundary condition[5]. The baffle causes diffraction effect when the complex array transmits and receives acoustic signals.

Some preliminary research work has been conducted on the influence of the baffle effect and mutual interactions among elements to the performance of the transducer array. Ref. [6] utilized the equivalent circuit principle to select appropriate 
tuning capacitors to control the vibration velocity of the transducer array. But this method was only an approximate method and difficult to be realized. Ref.[7,8] adopted the improved Helmholtz boundary integral equation method to calculate the acoustic radiation of transducer arrays, and analyzed the influence of the mutual radiation impedance on the vibration velocity and radiation directivity of the array. However, the above research work only analyzed that the baffle effect and mutual interactions greatly influenced the transmitting performance of the transducer array, and did not propose methods to reduce the influence so as to control the transmitting beampattern. We can utilize the boundary element model to calculate the radiated acoustic field of the array, and together with the equivalent circuit and optimization method calculate the driving voltage weighting vector to control the transmitting beampattern of the complex array.

When the receiving signals of the underwater acoustic transducer array are processed by beamforming considering the factors such as the mismatch error of each channel, the position perturbation of the elements, the scattering and shadowing of the baffle, and the mutual interactions among elements, the measured receiving array manifold vectors of the transducer array can be used to optimize the beampattern of the receiving signals. According to the acoustic reciprocity principle, the transmitting and receiving of the underwater acoustic transducer array is reciprocal[9-12]. When the transducer array transmits acoustic signals, the array may be influenced by the factors such as the baffle effect and mutual interactions among elements, the mismatch error of the channels, and so on. When it receives acoustic signals, the array may also be influenced by these factors. Thus, we can use the measured receiving array manifold vectors to calculate the driving voltage weighting vector of the underwater acoustic transducer array.

In this paper, two methods are proposed to obtain a lowsidelobe transmitting beampattern for complex arrays of underwater acoustic transducers. The boundary element model, transducer equivalent circuits, measured receiving array manifold vectors and optimization algorithm are used to calculate the driving voltage weighting vector of the complex array taking into account the baffle effect and mutual interactions among elements. This paper is structured as follows: Section 2 presents the materials and methods employed in this work. Section 3 describes the simulation and experimental results. Section 4 discusses the results obtained and Section 5 concludes this paper.

\section{MATERIALS AND METHODS}

This section introduces the equation of boundary element model and describes the acoustic field calculation method of transducer arrays using the model. It also presents the algorithm of boundary element model optimization method and measured receiving array manifold vector optimization method to obtain a low-sidelobe transmitting beampattern for complex arrays of underwater acoustic transducers.

\section{EQUATION OF BOUNDARY ELEMENT MODEL}

Assume that in the infinite uniform fluid medium, the smooth surface of the vibrating body is $S_{0}$, and $S_{1}$ is an infinite spherical surface which bounds $S_{0}$. The domain between $S_{0}$ and $S_{1}$ is $V$. The vibrating surface $S_{0}$ radiates sound outward the body to the fluid domain $V$. Then from the Helmholtz boundary integral equation we can get:

$$
\oiint_{S_{0}}\left(p(q) \frac{\partial G(q, Q)}{\partial n_{q}}-\frac{\partial p(q)}{\partial n_{q}} G(q, Q)\right) d S(q)=C(Q) P(Q)
$$

where $q$ is a point on the boundary surface $S_{0}$, and $Q$ is an arbitrary point in space. $n_{q}$ is the normal vector at $q$ on $S_{0}$ pointing toward the domain $V \cdot p$ is the sound pressure and $G(q, Q)$ is the Green's function in a three dimensional free space. The coefficient $C(Q)$ is equal to 1 for $Q$ in the acoustic domain $V$, equal to $1 / 2$ for $Q$ on the smooth boundary surface $S_{0}$, and equal to 0 for $Q$ outside the acoustic domain $V$.

The Green's function $G$ satisfies:

$$
G(r)=e^{-j k r} / 4 \pi r
$$

where $k$ is the wave number $(k=\omega / c$ with $\omega$ the angular frequency and ${ }^{c}$ the sound speed), $r=r(q, Q)$ is the distance between point $q$ and $Q$.

To solve the Helmholtz boundary integral equation numerically, we discretize the boundary $S_{0}$ into a series of boundary elements with $N$ nodes. The sound pressure at these nodes is denoted by $\left\{p_{q}\right\}$, and the normal vibration velocity is denoted by $\left\{v_{n}\right\}$. Choosing $M$ points in the radiated field, we denote the sound pressure of these points by $\left\{p_{Q}\right\}$. Then we can get:

$$
\left\{p_{Q}\right\}=[C]\left\{v_{n}\right\}
$$

Equations (3) shows that the sound pressure at any point in the radiated field of vibrating body can be obtained from the linear combination of the normal velocity vector, and the combination coefficients are defined by matrix $[C]$. The matrix is not dependent on the vibration velocity. It is only determined by the characteristics of the acoustic system, including the geometry of the vibrating surface, impedance boundary conditions, physical property of the acoustic medium (sound speed and fluid density), frequency and the location of the field points, etc. 


\section{PRINCIPLE OF BOUNDARY ELEMENT MODEL OPTIMIZATION METHOD}

The response vector $\mathbf{X}(\theta)$ in different directions on a certain distance from the center in the radiated acoustic field of the transducer array with a certain impedance boundary condition baffle can be obtained using the boundary element method by Eq.(3). Assume that the vibration velocity weighting vector of the transducer array is $\mathbf{W}_{V}$, then the sound pressure in the $\theta$ direction on a certain distance from the center of the array can be calculated as follows:

$$
p(\theta)=\mathbf{X}(\theta)^{T} \mathbf{W}_{V}
$$

Suppose that the driving voltage weighting vector of the transducer array is $\mathbf{W}_{E}$, then the vibration velocity weighting vector of the array can be calculated using the equivalent circuit principle $[9,11]$, which is:

$$
\mathbf{W}_{V}=n \mathbf{Z}^{-1} \mathbf{W}_{E}
$$

where $n$ is electromechanical transfer ratio of the transducers, and $\mathbf{Z}$ is the mutual impedance matrix of the array, which is:

$$
\mathbf{Z}=\left[\begin{array}{cccc}
Z_{m 0}+z_{11} & z_{12} & \cdots & z_{1 L} \\
z_{21} & Z_{m 0}+z_{22} & \cdots & z_{2 L} \\
\vdots & \vdots & \vdots & \vdots \\
z_{L 1} & z_{L 2} & \cdots & Z_{m 0}+z_{L L}
\end{array}\right]
$$

where $z_{\ddot{u}}$ is the self-radiation impedance of the ith transducer, and $z_{i j}$ is the mutual radiation impedance between the ith transducer and the jth transducer, and $Z_{m 0}$ is the mechanical impedance of the transducers in the array.

Substituting Eq. (5) into Eq. (4) produces:

$$
p(\theta)=n \mathbf{X}(\theta)^{T} \mathbf{Z}^{-1} \mathbf{W}_{E}
$$

Because the maximum amplitude of the driving voltage of the transducers in the array is limited, in order to obtain a low-sidelobe transmitting beampattern and a sound source level as high as possible, it needs to provide the largest pressure amplitude in the axial direction of the array when the maximum amplitude of the driving voltage weighting vectors keeps unchanged. That is, to make the maximum modulus of the driving voltage weighting vector to be the least, while producing the same amplitude of pressure in the axial direction of the array and at the same time imposing constraints on the sidelobe of the transmitting beampattern. As the driving voltage weighting vector to be calculated is the normalized vector, the coefficient $n$ in Eq. (7) can be left out of account. It leads to the following optimization problem:

$$
\left\{\begin{array}{cl}
\underset{\mathbf{w}_{E}}{\min } \mu & \\
\text { subject to } \quad & \mathbf{X}\left(\theta_{0}\right)^{T} \mathbf{Z}^{-1} \mathbf{W}_{E}=1 \\
& \left|\mathbf{W}_{E}(j)\right| \leq \mu, \quad j=1,2, \cdots, L \\
& \left|\mathbf{X}\left(\theta_{s}\right)^{T} \mathbf{Z}^{-1} \mathbf{W}_{E}\right| \leq \delta_{s}, \quad \forall \theta_{s} \in \Theta_{S L}
\end{array}\right.
$$

where $\mathbf{W}_{E}$ is the driving voltage weighting vector of the transducer array, $\mu$ is a nonnegative real variable, $L$ is the transducer number of the array, $\theta_{0}$ is the mainlobe direction, $\theta_{s}$ is the sidelobe direction, $\Theta_{S L}$ is the sidelobe regions which includes a number of directions and $\delta_{s}$ controls the sidelobe level of the transmitting beampattern, $\mathbf{X}(\theta)$ is the radiated acoustic pressure response vector in different directions, and $\mathbf{Z}$ is the mutual impedance matrix of the transducer array.

The optimization problem (8) can be solved by means of the optimization algorithm of the second-order cone programming[13]. Then, the required driving voltage weighting vector of the transducer array is calculated. The practical driving voltage exerted on the transducers in the array is obtained by normalizing the calculated weighting vector, in which the weighting vector is divided by the maximum modulus of the vector, and then multiplied by a voltage factor which must be less than the maximum voltage that can be exerted on the transducers of the array.

\section{PRINCIPLE OF MEASURED RECEIVING ARRAY MANIFOLD VECTOR OPTIMIZATION METHOD}

In the transducer array, the acoustical emission and receiving is reciprocal. The transmitting transducers may be affected by the other transducers and the baffle, and may be affected by the system error such as the channel mismatch among transducer and transducer, etc. And the receiving transducers are also affected by these factors. Then, we can calculate the driving voltage weighting vector with the optimization method utilizing the measured receiving array manifold vectors of the transducer array. Thus the calculation error of the weighting vectors can be reduced which is caused by the error of the theoretical boundary element model comparing to the practical system, since the measured receiving array manifold vectors can embody the error of the practical transducer array.

Suppose that the measured receiving array manifold vector of the transducer array in the $\theta$ direction is $\mathbf{V}(\theta)$. In order to obtain a low-sidelobe transmitting beampattern and a sound source level as high as possible, we make the maximum modulus of the driving voltage weighting vector to be the least, while producing the same amplitude of pressure in the axial direction and at the same time imposing constraints on the sidelobe of the transmitting beampattern, this is the following optimization problem: 


$$
\left\{\begin{array}{cc}
\min _{\mathbf{w}_{E}} \mu \\
\text { subject to } & \mathbf{V}\left(\theta_{0}\right)^{T} \mathbf{W}_{E}=1 \\
& \left|\mathbf{W}_{E}(j)\right| \leq \mu, \quad j=1,2, \cdots, L \\
& \left|\mathbf{V}\left(\theta_{s}\right)^{T} \mathbf{W}_{E}\right| \leq \delta_{s}, \quad \forall \theta_{s} \in \Theta_{S L}
\end{array}\right.
$$

where $\mathbf{W}_{E}$ is the driving-voltage weighting vector of the transducer array, $\mu$ is a nonnegative real variable, $L$ is the transducer number of the array, $\theta_{0}$ is the mainlobe direction, $\theta_{s}$ is the sidelobe direction, $\Theta_{S L}$ is the sidelobe regions which includes a number of directions and $\delta_{s}$ controls the sidelobe level of the transmitting beam pattern, $\mathbf{V}(\theta)$ is the measured receiving array manifold vectors in different directions.

As the same as above, we can use the second-order cone programming to solve the optimization problem (9) and obtain the required driving voltage weighting vector of the transducer array.

\section{RESULTS}

This section presents the simulation and experimental results of the methods proposed in this work.

\section{SIMULATION RESULTS}

To examine the effect of the boundary element model optimization method, we use the first transducer array. This array consists of 14 piston transducers. It is set in the middle arc line of a hemispherical baffle. The radius of the hemispherical baffle is $0.216 \mathrm{~m}$. The radiation surface of each piston transducer is rectangular with the length and $0.04 \mathrm{~m}$. The sound frequency is $12.5 \mathrm{kHz}$, and the wavelength is $0.12 \mathrm{~m}$. Two methods are used to calculate the driving voltage weighting vector of the transducer array. The first method is the conventional beamforming method in which the weighting vector is calculated by phase compensation according to the geometrical positions of the elements by the plane wave model without considering the baffle effect and mutual interactions among elements[14]. The other method is the boundary element model optimization method proposed in this paper, denoted by proposed method 1, which uses Eq. (8) to calculate the weighting vector of the array. Fig. 1 Fig.2 are respectively the radiated sound pressure amplitude and directivity of the 14-element array calculated by the boundary element method when the beam scan angle is $75^{\circ}$. The maximum pressure amplitude has been normalized to 1 . The solid line represents that the weighting vector is calculated by the boundary element model optimization method with the sidelobe level constrained to $-20 \mathrm{~dB}$. The dotted line represents that the weighting vector is calculated by the conventional beamforming method with the plane wave model. Fig. 1 Fig.2 shows that the proposed boundary element model optimization method in this paper can be used to control the sidelobe of the transmitting beampattern of the transducer array and obtain a low-sidelobe level. Using the proposed method 1, the highest sidelobe level of the beampattern is $-20 \mathrm{~dB}$. Using the conventional beamforming method, the highest sidelobe level is $-5.7 \mathrm{~dB}$. On the other hand, because the proposed method impose constraints on the sidelobe level of the beampattern comparing with the conventional beamforming method, the maximum pressure amplitude of the mainlobe may reduce. The proposed method can provide the largest pressure amplitude in the axial direction of the transducer array when the maximum amplitude of the driving voltage weighting vectors keeps unchanged, at the same time satisfying the sidelobe constraints. Corresponding to Fig. 1, using the proposed method 1 comparing with the conventional beamforming method, the maximum pressure amplitude does not reduce much, which is $1.67 \mathrm{~dB}$. It can be seen from above that the mutual interactions among elements and the baffle with a certain impedance boundary condition may greatly influence the radiated acoustic field of the transducer array. When the driving voltage weighting vector using the conventional beamforming method without considering the acoustic interactions, the radiation beampattern of the array may be distorted with high sidelobe level. However, the proposed method 1 can successfully take into account the acoustic interactions, and with reasonable optimization, a lowsidelobe beampattern and good transmitting performance can be obtained.

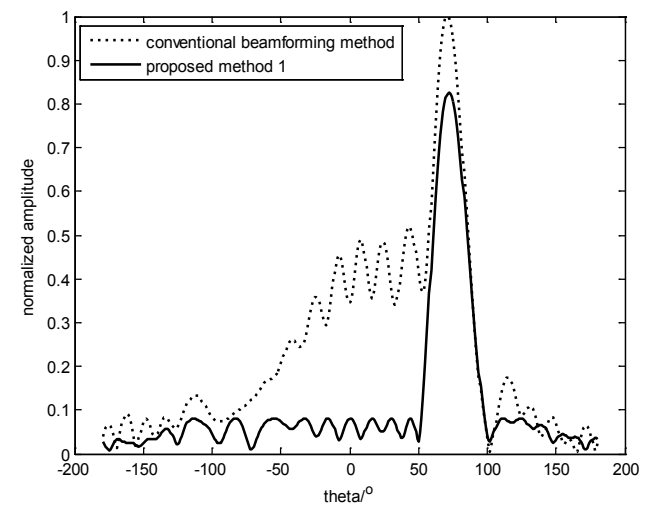

Fig. 1 . The radiated pressure amplitude of the array when the beam scan angle is $75^{\circ}$.

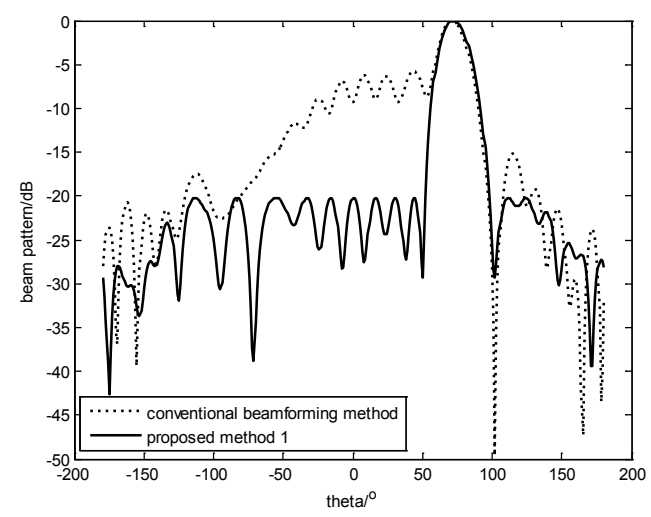

Fig. 2. The radiation directivity of the array when the beam scan angle is $75^{\circ}$. 
To examine the effect of measured receiving array manifold vector optimization method, we use the second transducer array, which is a little more complex than the first transducer array. This array consists of 27 piston transducers. It is set in the two arc lines of a hemispherical baffle. The 27-element array is divided into two rows. The first row is in the middle. The left side and the right side of the two rows are symmetric. The first row consists 14 transducers, and the second row consists 13 transducers. The hemispherical baffle and transducer elements are the same as those of the first 14-element array. The radius of the hemispherical baffle is $0.216 \mathrm{~m}$. The radiation surface of each piston transducer is rectangular with the length $0.04 \mathrm{~m}$. The sound frequency is $12.5 \mathrm{kHz}$, the sound speed underwater is $1500 \mathrm{~m} / \mathrm{s}$. An experiment has been carried out to measure the receiving array manifold vectors of the 27-element array in the anechoic water tank. The response of each element in the array to the plane-wave acoustic signals, including amplitude response and phase response, is measured in the range of $\pm 135^{\circ}$ from the main axial direction of the array with the interval $3^{\circ}$, thus, the receiving array manifold vectors of the array are obtained. Two methods are used to calculate the driving voltage weighting vector of the array. The first method is the conventional beamforming method. The other method is the measured receiving array manifold vector optimization method proposed in this paper, denoted by proposed method 2, which uses Eq. (9) to calculate the weighting vector of the transducer array. Fig. 3 Fig.4 are respectively the radiated sound pressure amplitude and directivity of the 27-element array calculated by simulation when the beam scan angle is $84^{\circ}$. The solid line represents that the weighting vector is calculated by the measured receiving array manifold vector optimization method in this paper with the sidelobe level constrained to $-20 \mathrm{~dB}$. The dotted line represents that the weighting vector is calculated by the conventional beamforming method with the plane wave model. Fig. 3 Fig. 4 show that the proposed measured receiving array manifold vector optimization method in this paper can also be used to control the sidelobe of the transmitting beam pattern of the transducer array and obtain a low-sidelobe level. Using the proposed method, the highest sidelobe level of the beam pattern by simulation is $-20 \mathrm{~dB}$. Using the conventional beamforming method, the highest sidelobe level is $-7.3 \mathrm{~dB}$. On the other hand, the proposed method 2 can also provide the largest pressure amplitude in the axial direction of the transducer array when the maximum amplitude of the driving voltage weighting vectors keeps unchanged, at the same time satisfying the sidelobe constraints. Corresponding to Fig. 3, using the proposed method 2 comparing with the conventional beamforming method, the maximum pressure amplitude does not reduce much, with the reduction amounts of $0.3 \mathrm{~dB}$. The simulation results show that the proposed method 2 can also be used to calculate the driving voltage weighting vector of the transducer array to obtain a low-sidelobe transmitting beampattern, and provide the largest pressure amplitude in the axial direction of the array when the maximum amplitude of the driving voltage weighting vectors keeps unchanged.

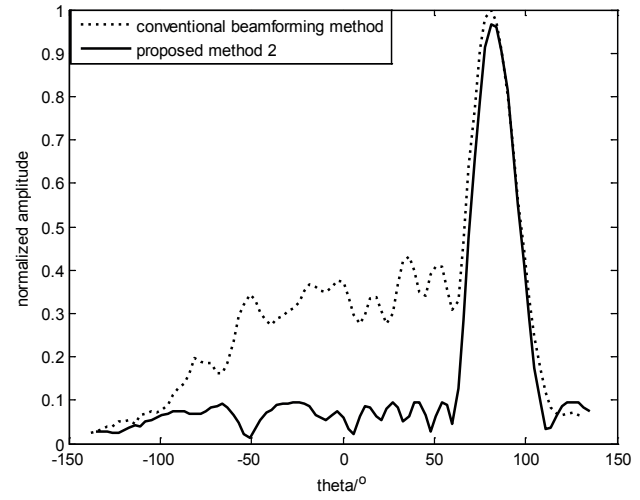

Fig. 3. The radiated pressure amplitude of the array when the beam scan angle is $84^{\circ}$

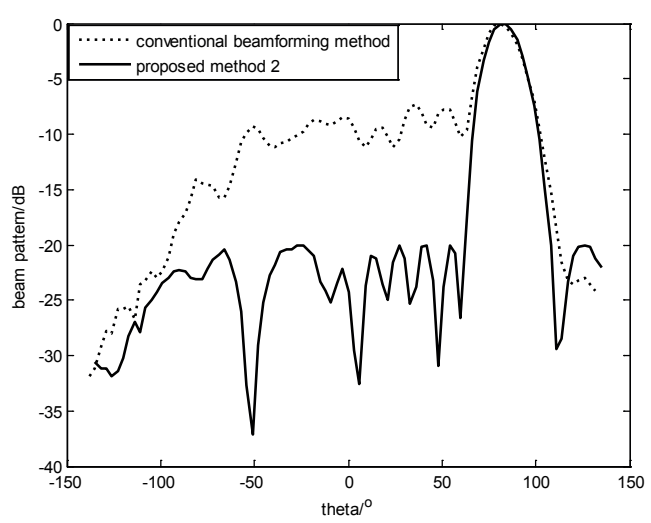

Fig. 4. The radiation directivity of the array when the beam scan angle is $84^{\circ}$.

\section{EXPERIMENTAL RESULTS}

An experiment has been conducted to measure the radiated acoustic pressure directivity of 14-element and 27-element array in the anechoic water tank. The working frequency is $12.5 \mathrm{kHz}$. The maximum driving voltage exerted on the transducer array is $4 \mathrm{~V}$, that is, the normalized driving voltage weighting vector calculated by Eqs. (8) and (9) is multiplied by the voltage factor $4 \mathrm{~V}$.

Fig. 5 Fig. 6 are respectively the measured radiated sound pressure amplitude and directivity of the 14-element array when the beam scan angle is $75^{\circ}$. The maximum pressure amplitude has been normalized to 1 . It can be found that the results measured in the experiment are consistent with the simulation results, which reveals that the boundary element model optimization method can calculate the driving voltage weighting vector of the transducer array to obtain a low-sidelobe transmitting beampattern. Using the proposed method 1, the highest sidelobe level of the measured beampattern is $-15.3 \mathrm{~dB}$ when the beam scan angle is $75^{\circ}$. Using the conventional beamforming method, the highest sidelobe level of the measured beampattern is $-5.9 \mathrm{~dB}$. It is shown that the highest sidelobe level reduce much using the propose method 1 comparing with the conventional beamforming method. There are some differences between 
the experimental results and the theoretical simulation results caused by the modeling error, calculation error and experiment error. On the other hand, just as the same in simulation computation, the proposed method 1 can provide the largest pressure amplitude in the axial direction of the transducer array when the maximum amplitude of the driving voltage weighting vectors keeps unchanged, and at the same time satisfying the sidelobe constraints. Corresponding to Fig. 5, by use of the proposed method 1 comparing with the conventional beamforming method, the maximum pressure amplitude does not reduce much, which is $1.72 \mathrm{~dB}$. It can be seen that the experimental results are consistent with the simulation results, which verifies that the proposed boundary element model optimization method is valid.

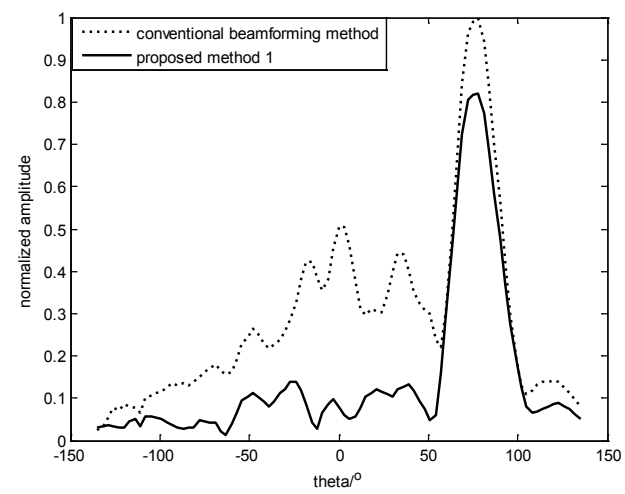

Fig. 5. The measured radiated pressure amplitude of the array when the beam scan angle is $75^{\circ}$

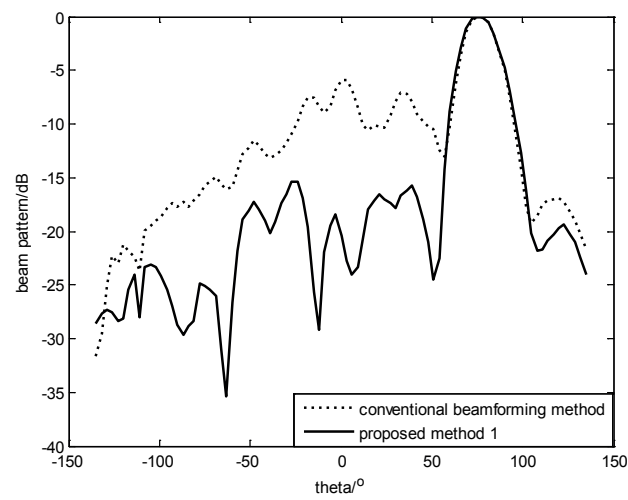

Fig. 6. The measured radiation directivity of the array when the beam scan angle is $75^{\circ}$.

Fig. 7 Fig. 8 are respectively the measured radiated sound pressure amplitude and directivity of the 27-element array when the beam scan angle is $84^{\circ}$. The solid line represents that the driving voltage weighting vector is calculated by the measured receiving array manifold vector optimization method with the sidelobe level constrained to -20dB. The dotted line represents that the weighting vector is calculated by the conventional beamforming method with the plane wave model. Fig. 7 Fig. 8 show that the results measured in the experiment are consistent with the simulation results, which reveal that the proposed method 2 can also be used to calculate the driving voltage weighting vector of the array to obtain a low-sidelobe transmitting beam pattern. Using the proposed method 2, the highest sidelobe level of the measured beam pattern is $-13.9 \mathrm{~dB}$ when the beam scan angle is $84^{\circ}$. Using the conventional beamforming method, the highest sidelobe level of the measured beam pattern is $-6.6 \mathrm{~dB}$. It is shown that the highest sidelobe level reduce much using the propose method 2 comparing with the conventional beamforming method. There are some differences between the experimental results and the theoretical simulation results because of the systematical error, the measurement error, and the error in the measured receiving array manifold vectors. The proposed method 2 can also provide the largest pressure amplitude in the axial direction of the transducer array when the maximum amplitude of the driving voltage weighting vectors keeps unchanged. Corresponding to Fig. 7, by use of the proposed method 2 comparing with the conventional beamforming method, the maximum pressure amplitude does not reduce much, with the reduction amounts of $0.29 \mathrm{~dB}$. It can be seen that the results measured in the experiment are greatly consistent with the simulation results, which verifies that the proposed measured receiving array manifold vector optimization method is also valid and practical.

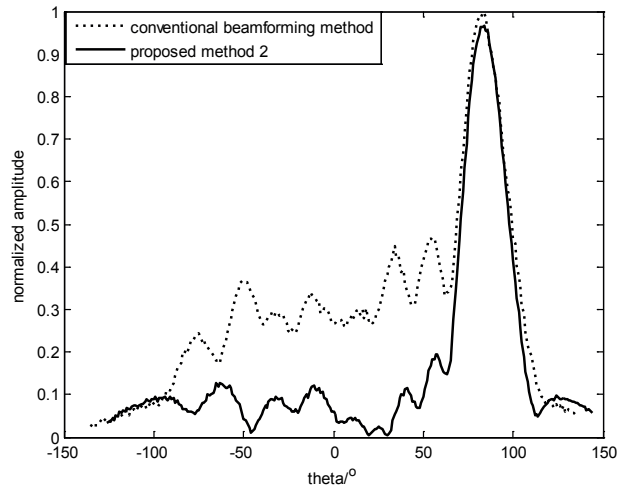

Fig.7. The measured radiated pressure amplitude of the array when the beam scan angle is $84^{\circ}$.

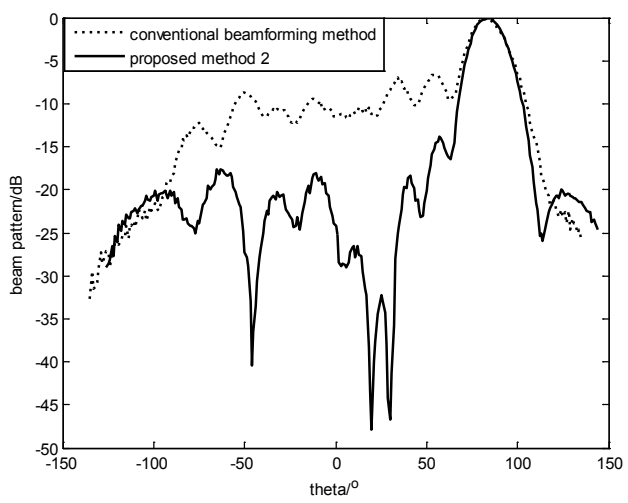

Fig. 8. The measured radiation directivity of the array when the beam scan angle is $84^{\circ}$. 


\section{DISCUSSION}

From the simulation and experimental results, we can see that both the boundary element model optimization method and the measured receiving array manifold vector optimization method are able to obtain a low-sidelobe transmitting beampattern for complex arrays of underwater acoustic transducers. However, each method has its own characteristics. The boundary element model optimization method is completely based on the theoretical calculation, which uses the boundary element model and the transducer equivalent circuits to calculate the driving voltage weighting vector of the acoustic array, and does not need the cost of experiment. The measured receiving array manifold vector optimization method needs to measure the array manifold vector of the acoustic array by experiment. But the effect of the measured receiving array manifold vector optimization method is better than that of the boundary element model optimization method, since it can achieve a more stable and reliable performance. The reason is that there is some error between the boundary element theoretical model and the practical system in the experiment, and the measured receiving array manifold vectors can embody this error of the practical transducer array.

\section{CONCLUSION}

In this paper, the methods are investigated to calculate the driving voltage weighting vector of complex acoustic arrays in underwater unmanned vehicles. Two methods are presented in order to obtain a low-sidelobe transmitting beampattern, which are respectively the boundary element model optimization method and the measured receiving array manifold vector optimization method. Computer simulation has been carried out for complex arrays to calculate the driving voltage weighting vector and transmitting beampatterns. The experiment has also been conducted to measure the radiated pressure amplitude and directivity of complex arrays in the anechoic water tank. The results show that the two methods are both able to obtain a lower sidelobe transmitting beampattern than the conventional beamforming method, and the source level for each transmitting beam is maximized in constraint of the maximum driving voltage of array elements being constant. Moreover, the effect of the measured receiving array manifold vector optimization method is better than that of the boundary element model optimization method, which can obtain a more stable performance and is more suitable for practical system application. The proposed methods are both able to improve the detecting and positioning capability of underwater unmanned vehicles.

\section{REFERENCES}

1. B. S. Liu, J. Y. Lei. The Principle of Underwater Acoustics (in Chinese). Harbin: Harbin Shipbuilding Engineering Institute Press, 1993. 6-56.

2. Jean-Noel Decarpigny. The design of low-frequency underwater acoustic projectors: present status and future trends. IEEE J Ocean Eng, 1991, 16(1): 107-122.

3. G. H. Du, Z. M. Zhu, X. F. Gong. Fundamentals of Acoustics (in Chinese). Nanjing: Nanjing University Press, 2001. 308-382.

4. J. Lee, I. Seo. Radiation power estimation for sonar transducer arrays considering acoustic interaction. Sens Actuat, 2001, 90: 1-6.

5. Christan Audoly. Some aspects of acoustic interactions in sonar transducer arrays. J.Acoust.Soc.Am., 1991, 89(3):1428-1433.

6. D. L. Carson.Diagosis and cure of erratic velocity distribution in sonar projector arrays. J Acoust Soc Am, 1962, 34(9): 1191-1195.

7. H. A. Schenck. Improved integral formulation for acoustic radiation problems. J.Acoust.Soc.Am., 1968, 44(1):41-58.

8. Tomoki Yokoyama,Mitsuru Henmi. Effects of mutual interactions on a phased transducer array. Jpn.J.Appl. Phys.,1998,37(5):3166-3171.

9. L. L. Foldy, H. Primakoff. A general theory of passive linear electroacoustic transducer and the electroacoustic reciprocity theorem. I. J.Acoust.Soc.Am.,1945, 17(2): 109-120.

10. R. J. Bobber. Diffraction constant of transdudcers. J.Acoust. Soc.Am.,1965, 37(4): 591-595.

11. W.S.Baidike. L.S. Fang(translation).Underwater acoustic system analysis. Beijing: Ocean Press, 1992: 39-58.

12. L. E. Kinsler. Fundamentals of Acoustics. New York: John Wiley \& Sons Inc, 2000. 390-406.

13. J.F.Sturn,Using SeDuMi 1.02, a MATLAB toolbox for optimization over symmetric cones, Opt.Meth. Softw.1999,11-12:625-653,.

14. W. H. Kummer. Basic array theory. Proc. IEEE, 1992, 80(1):127-139. 


\section{CONTACT WITH THE AUTHOR}

\section{Zhengyao He}

e-mail:hezhengyao@163.com

Institute of Acoustic Engineering

Northwestern Polytechnical University

Xi'an, 710072

China 